STRUCTURAL BIOLOGY

ISSN 2059-7983

Received 19 March 2021

Accepted 17 June 2021

Edited by G. Cingolani, Thomas Jefferson University, USA

Keywords: vancomycin resistance; X-ray crystallography; two-component systems; response regulators; Streptomyces coelicolor; full-length VanR

PDB references: $V_{a n} R_{S c}$, inactive, $7 / z 9$; activated, $7 \mathrm{lza}$

Supporting information: this article has supporting information at journals.iucr.org/d
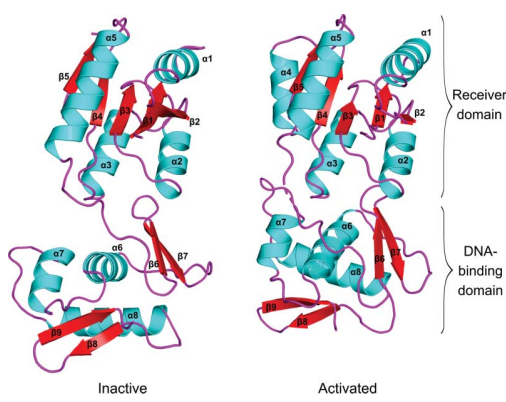

Activated

\section{Structures of full-length VanR from Streptomyces coelicolor in both the inactive and activated states}

\author{
Lina J. Maciunas, ${ }^{\text {a,b }}$ Nadia Porter, ${ }^{a, c}$ Paula J. Lee, ${ }^{a}$ Kushol Gupta ${ }^{d}$ and \\ Patrick J. Loll ${ }^{\mathrm{a} *}$
}

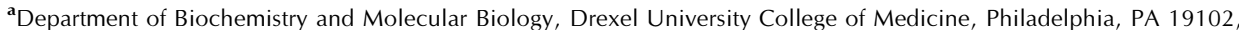
USA, 'braduate Program in Biochemistry, Drexel University College of Medicine, Philadelphia, PA 19102, USA, 'Summer Undergraduate Research Fellowship Program, Drexel University College of Medicine, Philadelphia, PA 19102, USA, and ${ }^{\mathbf{d}}$ Department of Biochemistry and Biophysics, University of Pennsylvania Perelman School of Medicine, Philadelphia, PA 19104, USA. *Correspondence e-mail: pjl28@drexel.edu

Vancomycin has historically been used as a last-resort treatment for serious bacterial infections. However, vancomycin resistance has become widespread in certain pathogens, presenting a serious threat to public health. Resistance to vancomycin is conferred by a suite of resistance genes, the expression of which is controlled by the VanR-VanS two-component system. VanR is the response regulator in this system; in the presence of vancomycin, VanR accepts a phosphoryl group from VanS, thereby activating VanR as a transcription factor and inducing expression of the resistance genes. This paper presents the X-ray crystal structures of full-length VanR from Streptomyces coelicolor in both the inactive and activated states at resolutions of 2.3 and $2.0 \AA$, respectively. Comparison of the two structures illustrates that phosphorylation of VanR is accompanied by a disorder-to-order transition of helix 4 , which lies within the receiver domain of the protein. This transition generates an interface that promotes dimerization of the receiver domain; dimerization in solution was verified using analytical ultracentrifugation. The inactive conformation of the protein does not appear intrinsically unable to bind DNA; rather, it is proposed that in the activated form DNA binding is enhanced by an avidity effect contributed by the receiver-domain dimerization.

\section{Introduction}

Two-component systems are critical to the survival of bacteria, mediating processes that include cell division, chemotaxis and antibiotic resistance. Each two-component system is composed of a sensor histidine kinase and a response regulator, which together coordinate an appropriate cellular response to an environmental stimulus. The kinase detects the stimulus and, in response, autophosphorylates on a histidine residue. This phosphoryl group is then transferred to an aspartate residue on the response regulator, thereby activating it (West \& Stock, 2001). Once activated, the response regulator produces an output that represents a suitable reaction to the stimulus. Depending on the response regulator, this output can involve binding to DNA, interacting with another protein, or expressing an enzymatic activity (Galperin, 2006).

VanR is the response regulator of the VanR-VanS twocomponent system, which controls the expression of a vancomycin-resistant phenotype in many bacteria, including soil microbes such as Streptomyces coelicolor as well as serious human pathogens such as vancomycin-resistant enterococci 
(VRE; Hong et al., 2008). VanR is composed of an N-terminal receiver domain, which contains the conserved aspartate that is the target for phosphorylation, and a C-terminal DNAbinding domain, which enables VanR to function as a transcription factor. Upon phosphorylation, VanR is thought to dimerize, bind to promoters within the vancomycinresistance operon, and activate transcription of the resistance genes (Arthur et al., 1992; Holman et al., 1994; Evers \& Courvalin, 1996; Depardieu et al., 2005). However, the structural transitions associated with VanR activation remain incompletely understood.

VanR belongs to the $\mathrm{OmpR} / \mathrm{PhoB}$ class of response regulators (Nguyen et al., 2015). This class of proteins has been the focus of extensive structural characterization, and structures are available of receiver domains in their inactive and activated states (Bachhawat et al., 2005; Toro-Roman, Mack et al., 2005; Toro-Roman, Wu et al., 2005), DNA-binding domains in their free and DNA-bound states (Blanco et al., 2002; Wang et al., 2007; He et al., 2016) and full-length proteins in either inactive or activated states (Buckler et al., 2002; Robinson et al., 2003; Nowak et al., 2006; Friedland et al., 2007; Narayanan et al., 2014; Lou et al., 2015). However, to the best of our knowledge, there are no full-length proteins in the OmpR/ PhoB class for which structures are known of both the inactive and activated forms. Indeed, only a few response regulators of any class have been crystallized in both forms, limiting our understanding of the mechanistic basis of activation.

In this paper, we present the structures of full-length VanR from Streptomyces coelicolor $\left(\operatorname{VanR}_{\mathrm{Sc}}\right)$ in its inactive and activated states. These represent the first reported structures of any VanR protein, as well as the first report of structures of an OmpR/PhoB-type response regulator in both activity states. Comparison of the two $\operatorname{VanR}_{\mathrm{Sc}}$ structures suggests that dimerization is the primary mechanism underlying activation.

\section{Materials and methods}

\subsection{Cloning, expression and purification}

The gene for VanR $\mathrm{Sc}_{\mathrm{Sc}}\left(\mathrm{NC}_{-}\right.$003888.3) was amplified from the genome of S. coelicolor M145 (ATCC BAA-471; Bentley et al., 2002) using forward primer $5^{\prime}$-AGA TTG GTG GCG GAA TGC GTG TGC TGA TTG TCG AG-3' ${ }^{\prime}$ and reverse primer 5'-GAG GAG AGT TTA GAC ATT ACT ATC CAC CGT CGC CGC C-3'. The amplified gene was subcloned into the in-house vector pETHSUL (Weeks et al., 2007), generating an expression construct for $\mathrm{VanR}_{\mathrm{Sc}}$ containing an $\mathrm{N}$-terminal $\mathrm{His}_{6}$-SUMO tag that could be cleaved with SUMO hydrolase. To facilitate SUMO cleavage, a glycine residue was inserted directly upstream of the first residue of $V a n R_{S c}$. Thus, the final purified VanR protein consists of residues 1-231 plus the additional $\mathrm{N}$-terminal glycine. The $\mathrm{VanR}_{\mathrm{Sc}}-\mathrm{SUMO}$ fusion protein was expressed in Escherichia coli BL21(DE3) cells, which were grown in 2.51 Ultra Yield flasks (Thomson Instrument Co., part No. 931136-B) in lysogeny broth (LB) with shaking at $225 \mathrm{rev} \mathrm{min}^{-1}$. Cultures were grown to midexponential phase at $37^{\circ} \mathrm{C}$, after which the temperature was reduced to $18^{\circ} \mathrm{C}$ and protein expression was induced with $0.2 \mathrm{~m} M$ isopropyl $\beta$-D-1-thiogalactopyranoside (IPTG) for $18 \mathrm{~h}$. The cells were harvested by centrifugation and the pellets were stored at $-80^{\circ} \mathrm{C}$ until further use.

Pelleted cells from 41 of growth culture were thawed and resuspended in IMAC buffer $A(300 \mathrm{~m} M \mathrm{NaCl}, 40 \mathrm{~m} M$ Tris $\mathrm{pH} 8,25 \mathrm{~m} M$ imidazole) containing $10 \mu \mathrm{g} \mathrm{ml}^{-1}$ DNase, $2 \mu \mathrm{g} \mathrm{ml}^{-1}$ RNase, $1 \mathrm{mM} \mathrm{MgCl}$ and Pierce EDTA-free protease-inhibitor tablets. The resuspended cells were lysed in a C5 Emulsiflex cell homogenizer (Avestin, Ottawa, Ontario, Canada) at 103-137 MPa. The lysate was clarified by centrifuging the sample at $208000 \mathrm{~g}$ for $1 \mathrm{~h}$. The resultant supernatant was syringe-filtered through a sterile $0.45 \mu \mathrm{m}$ filter and was then loaded onto a $5 \mathrm{ml}$ HiTrap IMAC-HP column (GE Healthcare) equilibrated in IMAC buffer $A$. The column was washed with $25 \mathrm{ml}$ IMAC buffer $A$ followed by $25 \mathrm{ml} 10 \%$ IMAC buffer $B(300 \mathrm{~m} M \mathrm{NaCl}, 40 \mathrm{~m} M$ Tris $\mathrm{pH} 8,350 \mathrm{~m} M$ imidazole). The protein was eluted with a $25 \mathrm{ml}$ gradient from $10 \%$ to $100 \%$ IMAC buffer $B$. Fractions containing the fusion protein were pooled and the recombinant yeast SUMO hydrolase dtUD1 (Weeks et al., 2007) was added to the sample at a final concentration of $5 \mu \mathrm{g} \mathrm{m}{ }^{-1}$. The sample was dialyzed overnight against IMAC buffer $A$ lacking imidazole using $3.5 \mathrm{kDa}$ molecular-weight cutoff SnakeSkin dialysis tubing (Thermo Fisher Scientific, catalog No. 88244). The dialysate was passed over the IMAC column re-equilibrated in IMAC buffer $A$ to capture the His-SUMO fusion partner. The flowthrough containing $V_{a n R} R_{S c}$ was collected, concentrated and injected onto a Sephacryl S200 size-exclusion column (GE Healthcare) equilibrated in IMAC buffer $A$ lacking imidazole. The peak fractions were collected, concentrated and filtered through a $0.22 \mu \mathrm{m}$ filter. The concentration was determined from the $A_{280}$ using the calculated extinction coefficient $\varepsilon=$ $14440 \mathrm{M}^{-1} \mathrm{~cm}^{-1}$.

\subsection{Analytical ultracentrifugation}

Sedimentation-velocity analytical ultracentrifugation experiments were performed at $20^{\circ} \mathrm{C}$ with an XL-A analytical ultracentrifuge (Beckman-Coulter, Brea, California, USA) and a TiAn60 rotor with two-channel charcoal-filled Epon centerpieces and quartz windows. The $\mathrm{VanR}_{\mathrm{Sc}}$ protein was dissolved in $50 \mathrm{~m} M$ Tris pH 7.5, $50 \mathrm{~m} M \mathrm{NaCl}, 10 \mathrm{~m} M \mathrm{MgCl}_{2}$ with or without $5 \mathrm{mM} \mathrm{BeSO}_{4}$ and $35 \mathrm{~m} M \mathrm{NaF}$. Data were collected with detection at $280 \mathrm{~nm}$. Complete sedimentation-

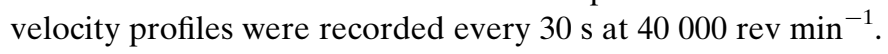
Data were fitted using the $c(s)$ or $c\left(s, f \mid f_{0}\right)$ distribution implementations of the Lamm equation as implemented in SEDFIT (Schuck, 2000) and corrected for $S_{20, w}$. Direct fitting of association models was performed using SEDPHAT (Vistica et al., 2004).

Sedimentation-equilibrium analytical ultracentrifugation data were collected at $4^{\circ} \mathrm{C}$ with detection at $280 \mathrm{~nm}$ and a TiAn60 rotor with six-channel charcoal-filled Epon centerpieces and quartz windows at three sample concentrations at 18000,20000 and $22000 \mathrm{rev} \mathrm{min}^{-1}$. Analyses were carried out using global fits to data acquired at multiple speeds at four 
Table 1

Data-collection and refinement statistics.

Values in parentheses are for the highest resolution shell.

\begin{tabular}{|c|c|c|}
\hline & Inactive (PDB entry 7lz9) & Activated (PDB entry 7lza) \\
\hline \multicolumn{3}{|l|}{ Data-collection statistics } \\
\hline Diffraction source & $\begin{array}{l}\text { Beamline 17-ID-1 (AMX), } \\
\text { NSLS-II }\end{array}$ & $\begin{array}{l}\text { Beamline 17-ID-1 (AMX), } \\
\text { NSLS-II }\end{array}$ \\
\hline Wavelength $(\AA)$ & 0.920089 & 0.920091 \\
\hline Temperature $(\mathrm{K})$ & 100 & 100 \\
\hline Detector & EIGER 9M & EIGER 9M \\
\hline Resolution range $(\AA)$ & $32.75-2.30(2.38-2.30)$ & $28.85-2.03(2.10-2.03)$ \\
\hline Space group & $P 6_{5} 22$ & $P 6_{5} 22$ \\
\hline$a, b, c(\AA)$ & $74.38,74.38,138.23$ & $97.37,97.37,118.65$ \\
\hline$\alpha, \beta, \gamma\left({ }^{\circ}\right)$ & $90.0,90.0,120.0$ & $90.0,90.0,120.0$ \\
\hline Total No. of observations & 27079 (24618) & $94478(8396)$ \\
\hline No. of unique reflections & $10592(1033)$ & 21574 (1981) \\
\hline Average multiplicity & $25.6(23.8)$ & $4.4(4.2)$ \\
\hline Completeness (\%) & 99.5 (98.9) & $97.5(91.8)$ \\
\hline Mean $I / \sigma(I)$ & $10.9(1.9)$ & $15.3(3.5)$ \\
\hline Estimated Wilson $B$ factor $\left(\AA^{2}\right)$ & 33.8 & 42.4 \\
\hline$R_{\text {merge }} \dagger$ & $0.246(1.953)$ & $0.052(0.406)$ \\
\hline$R_{\text {meas }} \ddagger$ & $0.251(1.996)$ & $0.059(0.463)$ \\
\hline$R_{\text {p.i.m. }} \S$ & $0.049(0.404)$ & $0.028(0.145)$ \\
\hline $\mathrm{CC}_{1 / 2}$ & $0.998(0.696)$ & $0.998(0.916)$ \\
\hline \multicolumn{3}{|l|}{ Refinement and model statistics } \\
\hline Resolution range $(\AA)$ & $32.75-2.30(2.38-2.30)$ & $28.85-2.03(2.10-2.03)$ \\
\hline No. of reflections used & $10062(1033)$ & $21562(1978)$ \\
\hline Reflections used for $R_{\text {free }}$ & $529(52)$ & $1077(98)$ \\
\hline$R_{\text {work }}$ & $0.202(0.268)$ & $0.182(0.198)$ \\
\hline$R_{\text {free }}$ & $0.256(0.339)$ & $0.222(0.252)$ \\
\hline Solvent content (\%) & 44 & 62 \\
\hline \multicolumn{3}{|l|}{ No. of non-H atoms } \\
\hline Protein & 1594 & 1685 \\
\hline Water & 28 & 184 \\
\hline $\mathrm{Mg}^{2+}$ & 1 & 1 \\
\hline $\mathrm{BeF}_{3}^{-}$ & - & 1 \\
\hline Average $B$ value $\left(\AA^{2}\right)$ & 43.0 & 41.4 \\
\hline \multicolumn{3}{|l|}{ R.m.s. deviations from ideality } \\
\hline Bond lengths $(\AA)$ & 0.003 & 0.01 \\
\hline Angles $\left({ }^{\circ}\right)$ & 0.59 & 0.85 \\
\hline \multicolumn{3}{|c|}{ Residue distribution in Ramachandran plot } \\
\hline Most favored region (\%) & 97.6 & 97.2 \\
\hline Allowed (\%) & 2.4 & 2.8 \\
\hline Outliers (\%) & 0.0 & 0.0 \\
\hline Clashscore & 1.86 & 3.52 \\
\hline
\end{tabular}

$\dagger R_{\text {merge }}=\sum_{h k l} \sum_{i}\left|I_{i}(h k l)-\langle I(h k l)\rangle\right| / \sum_{h k l} \sum_{i} I_{i}(h k l)$, where $I_{i}(h k l)$ is the $i$ th measurement of reflection $h k l . \quad \$ R_{\text {meas }}$ (or redundancy-independent $\left.R_{\text {merge }}\right)=\sum_{h k l}[N(h k l) /[N(h k l)-1]\}^{1 / 2} \sum_{i}\left|I_{i}(h k l)-\langle I(h k l)\rangle\right| / \sum_{h k l} \sum_{i} I_{i}(h k l)$, where $I_{\mathrm{i}}(h k l)$ is the $i$ th measurement and $N(h k l)$ is the redundancy of each unique reflection $h k l$ (Diederichs \& Karplus, 1997). $\& R_{\text {p.i.m. }}=\sum_{h k l}\{1 /[N(h k l)-1]\}^{1 / 2} \sum_{i}\left|I_{i}(h k l)-\langle I(h k l)\rangle\right| / \sum_{h k l} \sum_{i} I_{i}(h k l)$, where $I_{i}(h k l)$ is the $i$ th measurement and $N(h k l)$ is the redundancy of each unique reflection $h k l$ (Weiss, 2001). $\mathrm{CC}_{1 / 2}$ is the correlation coefficient between two randomly chosen half data sets (Karplus \& Diederichs, 2012).

concentrations with strict mass conservation using SEDPHAT (Vistica et al., 2004). Error estimates for equilibrium constants and fit masses were determined from a 1000-iteration Monte Carlo simulation.

The partial specific volume $(\bar{v})$, solvent density $(\rho)$ and viscosity $(\eta)$ were derived from the chemical composition by SEDNTERP (Laue et al., 1992). Figures were created using GUSSI (Brautigam, 2015).

\subsection{Crystallization and X-ray data collection}

The inactive state of $V_{a n R} R_{S c}$ was crystallized by microbatch under Al's Oil (Hampton Research, catalog No. HR3-413; Chayen et al., 1992; D'Arcy et al., 1996). Freshly purified protein was dialyzed against $500 \mathrm{~m} M \mathrm{NaCl}, 5 \mathrm{~m} M \mathrm{MgCl}_{2}$,
$10 \%(v / v)$ glycerol, $40 \mathrm{~m} M$ Tris $\mathrm{pH} 8$ and concentrated to $\sim 11.3 \mathrm{mg} \mathrm{ml}^{-1}$. The protein solution $(0.5 \mu \mathrm{l})$ was combined with an equal volume of crystallization condition $\mathrm{C} 1$ from Rigaku's Wizard Classic 1 and 2 screens [0.2 $\mathrm{M} \mathrm{MgCl}_{2}$, $0.1 M$ Tris $\mathrm{pH} 8.5,30 \%(w / v)$ PEG 400] using an Oryx6 Robot (Douglas Instruments, Berkshire, UK). Crystallization trays were incubated at $4{ }^{\circ} \mathrm{C}$ and thin needle-like crystals appeared within three weeks. Crystals were harvested without additional cryoprotectant and were flash-cooled by plunging into liquid nitrogen.

Crystals of the activated state of $V_{a n} R_{S c}$ were prepared by co-crystallizing freshly prepared $V_{a n R}$ with beryllium fluoride $\left(\mathrm{BeF}_{3}^{-}\right)$by microbatch under oil. The protein (in IMAC buffer $A$ lacking imidazole) was concentrated to $11.3 \mathrm{mg} \mathrm{ml}^{-1}$. A $10 \times$ $\mathrm{BeF}_{3}^{-}$stock solution was prepared consisting of $50 \mathrm{mM} \mathrm{BeSO}_{4}, 350 \mathrm{~m} M$ $\mathrm{NaF}, 70 \mathrm{~m} M \mathrm{MgCl}_{2}$. The $\mathrm{BeF}_{3}^{-}$stock was combined with the protein in a $1: 10(v: v)$ ratio, resulting in a final protein concentration of $\sim 10.2 \mathrm{mg} \mathrm{ml}^{-1}$. $0.5 \mu \mathrm{l}$ of the $\operatorname{VanR}_{\mathrm{Sc}}-\mathrm{BeF}_{3}^{-}$mixture was combined with $0.5 \mu \mathrm{l}$ crystallization condition F5 from Rigaku's Wizard Classic 1 and 2 screens $(2.5 \mathrm{M} \mathrm{NaCl}$, $0.1 M$ Tris $\mathrm{pH} 7,0.2 M \mathrm{MgCl}_{2}$ ). The crystallization tray was incubated at room temperature and rod-like crystals grew after three days. The crystals were dragged through the oil as a cryoprotectant and then plunged into liquid nitrogen.

Diffraction data for both crystal forms were measured on the AMX beamline of the National Synchrotron Light Source II (NSLS-II). Data-collection details are summarized in Table 1.

\subsection{Structure determination and refinement}

The data collected from crystals of VanR $\mathrm{R}_{\mathrm{Sc}}$ in both activity states were processed by $X D S$ and scaled with XSCALE (Kabsch, 2010). The structure of the activated $\operatorname{VanR}_{\mathrm{Sc}}$ was determined by molecular replacement with Phaser in Phenix (Zwart et al., 2008; Liebschner et al., 2019) utilizing two probes. The probes were chosen using BLAST searches employing the sequences of the $\operatorname{VanR}_{\mathrm{sc}}$ receiver and DNAbinding domains; residues 19-119 from PDB entry 5uic (Milton et al., 2017) were used to spatially orient the receiver domain and residues 134-220 from PDB entry $1 \mathrm{kgs}$ (Buckler 
et al., 2002) were used to orient the DNA-binding domain. The structure of the inactive $\operatorname{VanR}_{\mathrm{Sc}}$ was determined by molecular replacement using the two domains of the activated protein as probes. Models of the inactive $V_{a n R}$ and activated $V_{\mathrm{San}} \mathrm{R}_{\mathrm{Sc}}$ were built using the AutoBuild function in Phenix. These models were iteratively adjusted in Coot (Emsley et al., 2010) and refined in Phenix. The quality of the final models was assessed by both MolProbity (Chen et al., 2010) and the $R_{\text {free }}$ value. The $R_{\text {free }}$ value was based on $5 \%$ of the total reflections chosen at random prior to refinement. The final refinement statistics are shown in Table 1. Coordinates and structure factors were deposited with the Protein Data Bank (PDB entries $7 \mathrm{lz} 9$ and $7 \mathrm{lza}$ for the inactive and activated proteins, respectively). Raw diffraction images are available from the Zenodo repository (https://www.zenodo.org) using the following digital object identifiers: https://doi.org/10.5281/ zenodo.4594513 for inactive $\operatorname{VanR}_{\mathrm{Sc}}$ and https://doi.org/ 10.5281/zenodo.4593691 for activated VanR $R_{\text {Sc }}$.

All figures were made using PyMOL (version 2.3; Schrödinger). Interdomain interfaces were identified using both AREAIMOL within CCP4 version 7.0 (Lee \& Richards, 1971; Saff \& Kuijlaars, 1997; Winn et al., 2011) and the PISA web server (Krissinel \& Henrick, 2007). TM-align was used for the superposition of analogous domains (Zhang \& Skolnick, 2005). HELANAL-Plus was used to calculate helix axes (Kumar \& Bansal, 2012).

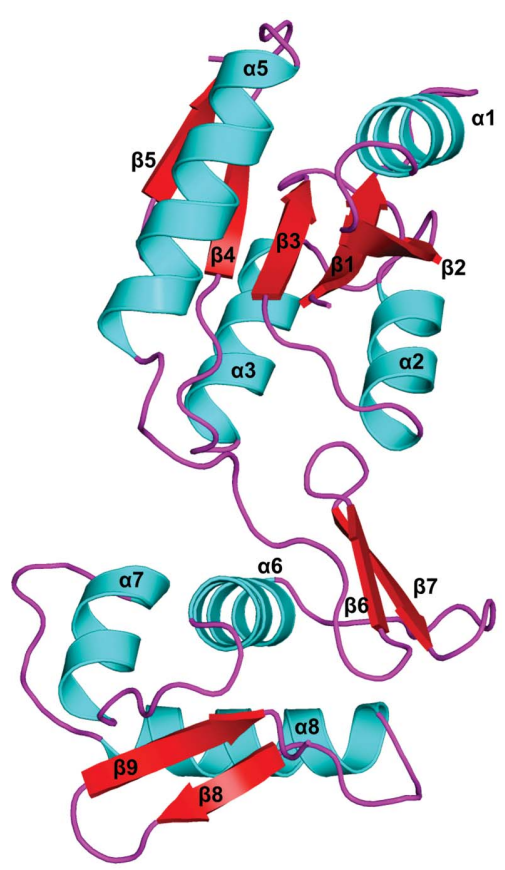

Inactive

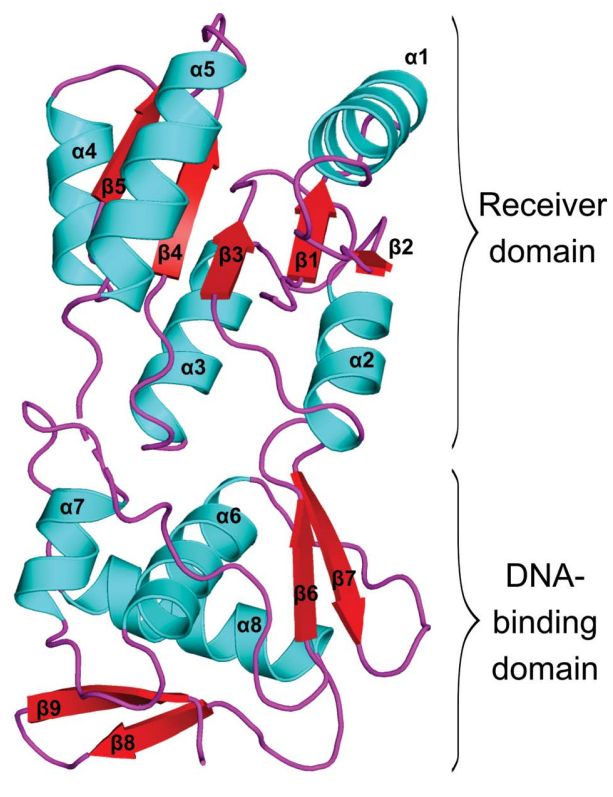

Activated
Figure 1

Crystal structures of full-length $\operatorname{VanR}_{\mathrm{Sc}}$ in the inactive and activated states. The receiver and DNAbinding domains are indicated. Secondary structures are colored as follows: $\beta$-strands, red; $\alpha$-helices, cyan; loops, magenta. Numbering is shown for $\alpha$-helices and $\beta$-strands. Helix $\alpha 4$ is absent from the inactive $V_{a n} R_{S c}$ structure, reflecting presumptive disorder. The loop connecting the receiver and DNA-binding domains is fully ordered in the inactive state, but is disordered at His121 in the activated state (indicated by dashes in the right-hand structure). Stereo versions of both panels can be found in Supplementary Fig. S1.

\section{Results}

\subsection{Structure determination}

Full-length recombinant $\operatorname{VanR}_{\mathrm{Sc}}$ was produced in E. coli and purified by subtractive immobilized metal-ion chromatography and gel filtration. The activated state was generated by treating the protein with beryllium fluoride, which has proven to act as a faithful mimic of aspartate phosphorylation in a variety of response regulators (Wemmer \& Kern, 2005). X-ray crystal structures were determined for the inactive and activated states at resolutions of 2.3 and $2.0 \AA$, respectively. Both activity states crystallized in the same $P 6_{5} 22$ space group but with different unit-cell dimensions; in both crystal forms the asymmetric unit contained a monomer (Fig. 1). For both conformational states, no electron density was observed for the 12 C-terminal residues (221-232), suggesting a highly flexible C-terminal tail. Details of the structure determination and refinement are given in Table 1.

\subsection{Overall description of the structures}

The receiver domains of many response regulators have been crystallized in the inactive and activated states, and the receiver domain of $V_{a n R} R_{S c}$ is very similar to those seen previously, with root-mean-square deviations (r.m.s.d.s) for $\mathrm{C}^{\alpha}$-atom positions ranging from 1.1 to $2.0 \AA$ (Supplementary Table S1; Stock et al., 1989; Robinson et al., 2000). The domain adopts an $\alpha / \beta$-sandwich fold composed of a central five-stranded parallel $\beta$-sheet with a 2-1-3-4-5 topology surrounded by three $\alpha$-helices on one side $(\alpha 2, \alpha 3$ and $\alpha 4)$ and two $\alpha$-helices on the other ( $\alpha 1$ and $\alpha 5)$.

As is true for other members of the OmpR/PhoB class of response regulators, the DNA-binding domain of VanR $_{S c}$ adopts a winged helix-turnhelix motif composed of three $\alpha$-helices $(\alpha 6, \alpha 7$ and $\alpha 8)$ followed by a C-terminal $\beta$-hairpin (Martínez-Hackert \& Stock, 1997). $\alpha 8$ is also referred to as the recognition helix, and is expected to bind within the major groove of DNA, making specific contacts with bases, sugars and the phosphate backbone of DNA. In addition to the winged-helix motif, DNA-binding domains of the OmpR/PhoB class typically contain a four-stranded antiparallel $\beta$-sheet upstream of the winged helix-turnhelix. In $V_{a n R}$, this $\beta$-sheet contains only two strands, along with the possible vestige of a third (Fig. 1). The lack of a full four-stranded $\beta$-sheet is uncommon but not unprecedented; for example, the DNA-binding domain of PmrA contains a sheet with only three antiparallel $\beta$-strands (Lou et al., 2015). In spite of 
the difference in this sheet region, the DNA-binding domain of VanR $R_{\mathrm{Sc}}$ is very similar overall to those of other OmpR/ PhoB response regulators, with r.m.s.d.s for $\mathrm{C}^{\alpha}$ atoms ranging from 1.3 to $2.7 \AA$ (Supplementary Table S2).

In OmpR/PhoB response regulators, the length of the linker connecting the receiver and DNA-binding domains ranges from five to 21 amino acids (Martínez-Hackert \& Stock, 1997). In $V_{a n R} R_{S c}$ the linker contains 11 residues; in addition, in the activated state, partial unwinding of the C-terminus of $\alpha 5$ extends the linker length by three residues. The functional significance of this length difference is unclear, but it may provide additional flexibility that allows optimal positioning of the DNA-binding domains upon their DNA target. While one might expect long linkers such as those in the $\operatorname{VanR}_{\mathrm{Sc}}$ structures to be highly flexible, the linkers are well ordered in the crystals of both activity states. In the structure of inactive $V_{a n R}$ the linkers from two adjacent molecules in the crystal lattice associate in an antiparallel manner, leading to the formation of a symmetric pair of hydrogen bonds between the carbonyl O atom of Arg123 and the amide proton of Arg123' of the symmetry mate, along with a similarly symmetric pair of hydrogen bonds between the side chain of $\operatorname{Arg} 123$ and the carbonyl $\mathrm{O}$ atom of Pro124'. In the activated VanR $\mathrm{Sc}_{\mathrm{sc}}$ structure the linker does not participate in any crystal contacts and yet remains almost completely ordered, with the exception of a single chain break at His121.

\subsection{Comparison of the receiver-domain structure with those} of other response regulators

The active site of the activated form of $V_{a n R_{S c}}$ resembles those found in other activated receiver-domain structures (Yan et al., 1999). The active site centers around the highly conserved receiver of the phosphoryl group, Asp51, which is located at the end of $\beta 3$. Similar to what is seen in other activated receiver-domain structures, the $\mathrm{BeF}_{3}^{-}$ion is coordinated by Asp51, together with a magnesium ion, to form a phosphomimetic (Fig. $2 a$ and $2 b$ ). The Be atom is bound to one of the carboxylate $\mathrm{O}$ atoms of Asp51, while the $\mathrm{F}$ atoms interact with the side chains of Thr79 and Lys101 and with the magnesium ion. The magnesium displays octahedral coordination, with its ligands including an $\mathrm{F}$ atom, the side chains of Asp51 and Asp8, the backbone carbonyl O atom of Asp53 and two water molecules. These water molecules interact with each other as well as with the side chains of Asp51, Glu7, Asp8 and Lys101, making them integral components in a network of hydrogen bonds that spans the active site.

Once activated, the receiver domains of the $\mathrm{OmpR} / \mathrm{PhoB}$ family assemble into twofold-symmetric dimers, with the $\alpha 4-$ $\beta 5-\alpha 5$ surface forming the dimer interface (Toro-Roman, $\mathrm{Wu}$ et al., 2005). For activated $\operatorname{VanR}_{\mathrm{Sc}}$, the crystal asymmetric unit only contains a monomer, but a dimer with the expected interface is formed by crystal symmetry (Fig. $2 c$ ). The $\alpha 4-\beta 5-$ $\alpha 5$ interface buries $846 \AA^{2}$ of surface area, with the fraction of atoms completely buried $\left(f_{\mathrm{BU}}\right)$ equal to 0.34 ; both of these values are consistent with this interface being biologically relevant (Ponstingl et al., 2000; Janin et al., 2007). In contrast, in the crystals of the inactive form of $V_{a n} R_{S c}$ none of the lattice contacts appear to correspond to biologically meaningful interfaces.

The $\alpha 4-\beta 5-\alpha 5$ dimer is specific to the $\mathrm{OmpR} / \mathrm{PhoB}$ class of response regulators, and relies on a set of conserved interactions that are unique to this class (Toro-Roman, Mack et al., 2005). These include hydrophobic interactions formed at the outer edges of the contact surface and polar interactions that stabilize the core of the interface. In $\operatorname{VanR}_{\mathrm{sc}}$ the hydrophobic interactions involve Ala88 and Phe91, which lie on $\alpha 4$ of one protomer, and Leu110, which is located on $\alpha 5$ of the facing protomer (Fig. 2c). The polar interactions involve five buried contacts across the dimer interface, which are made between the following pairs of residues: Glu107/Lys87, Asp97/Arg111, Asp96 (main chain)/Arg118, Asp96 (side chain)/Arg117 and Tyr98/Arg111 (Fig. 2d). Interactions involving the first three of these interacting pairs are highly conserved in $\mathrm{OmpR} / \mathrm{PhoB}$ response regulators, while interactions involving the fourth are seen in some but not all members of the family (Fig. 2e). The fifth interaction (Tyr98-Arg111) is atypical and appears to replace an interaction found in most family members, but not in VanR $R_{\mathrm{Sc}}$. Normally, OmpR/PhoB response regulators contain a salt bridge between a glutamate at the C-terminus of $\alpha 4$ and an arginine in $\alpha 5$; in $V_{a n R_{S c}}$ the corresponding residues are 92 and 113 . However, $\operatorname{VanR}_{\mathrm{Sc}}$ has a glycine at residue 92 , rather than a glutamate, and thus instead of the typical Glu-Arg salt bridge it forms an alternative polar contact between the backbone carbonyl of Tyr98 and the side chain of Arg111 (Fig. 2e). Despite this difference, the overall pattern of interactions within the dimer interface and the active site closely corresponds to those found in other activated response regulators, supporting the assumption that the $\mathrm{VanR}_{\mathrm{Sc}}-\mathrm{BeF}_{3}^{-}$ complex closely mimics the conformation of the phosphorylated protein.

\subsection{Experimental confirmation of oligomer formation}

To test whether dimerization accompanies $\operatorname{VanR}_{\mathrm{Sc}}$ phosphorylation in solution, we used analytical ultracentrifugation to probe the oligomerization state of the protein in the presence and absence of beryllium fluoride. In the absence of $\mathrm{BeF}_{3}^{-}$, sedimentation-velocity analysis revealed that the protein is largely present as a monomer, with small amounts of a more rapidly sedimenting species that might correspond to a compact dimer (Figs. $3 a$ and $3 b$ ). Upon the addition of $\mathrm{BeF}_{3}^{-}$ the profile shifts toward higher molecular-weight species, including a dimer and a putative tetramer. The polydispersity of the sample also increases in the presence of $\mathrm{BeF}_{3}^{-}$, with a particular increase in more extended species (Supplementary Fig. S4). We also examined the behavior of the protein in a sedimentation-equilibrium analysis; again, the addition of $\mathrm{BeF}_{3}^{-}$was accompanied by a clear shift toward higher-order species (Fig. 3c).

A variety of models were used to fit the centrifugation data. For the sedimentation-velocity experiments performed in the absence of $\mathrm{BeF}_{3}^{-}$, the data were best fitted by a very weak monomer-dimer equilibrium model, with a $K_{\mathrm{d}}$ of $>1 \mathrm{~m} M$ 
(Supplementary Table S3). The sedimentation-equilibrium data measured in the absence of $\mathrm{BeF}_{3}^{-}$are also well described by a weak monomer-dimer equilibrium model, consistent with the sedimentation-velocity results; however, a somewhat lower estimate of $K_{\mathrm{d}}=37 \mu M$ was obtained (Supplementary Table S4). This may reflect differences in experimental conditions: the equilibrium experiments were performed at $4^{\circ} \mathrm{C}$, while the velocity experiments were conducted at $20^{\circ} \mathrm{C}$, and this temperature dependence might reflect a contribution from hydrophobic interactions during dimerization. In any case, however, it is evident that the monomer is the predominant species for the unphosphorylated protein.

In the presence of $\mathrm{BeF}_{3}^{-}$, the velocity data are well described by a monomer-dimer-tetramer equilibrium model,

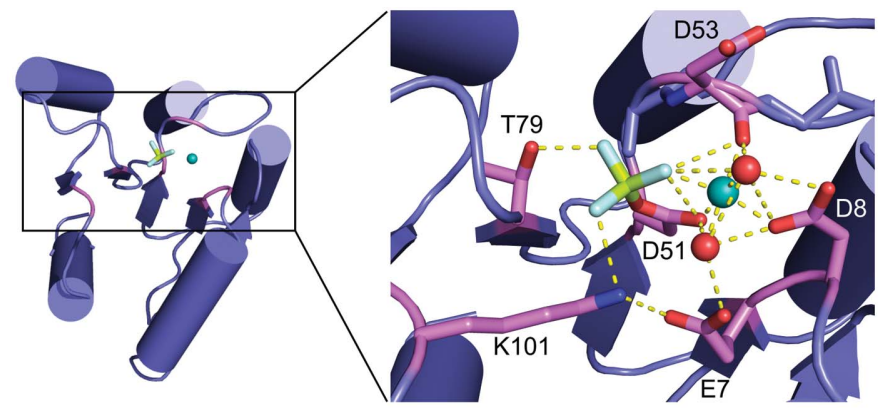

$(a)$

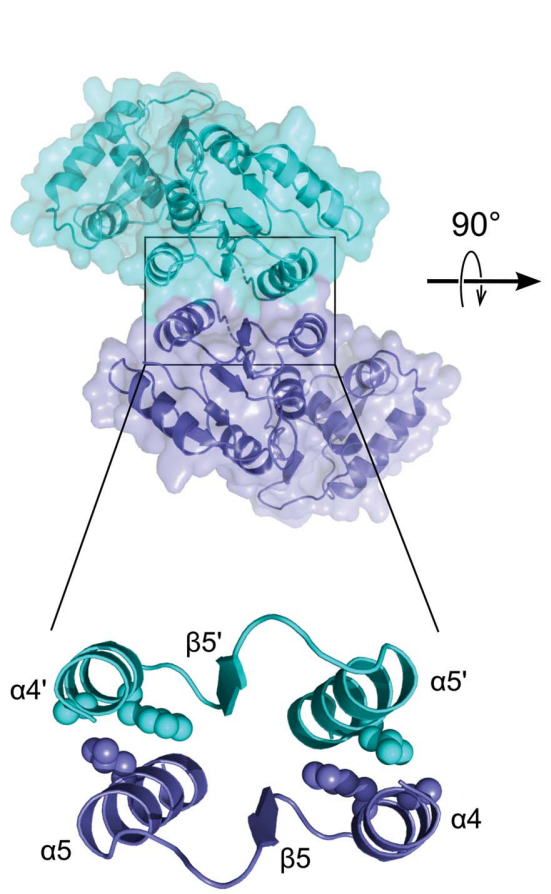

(c)
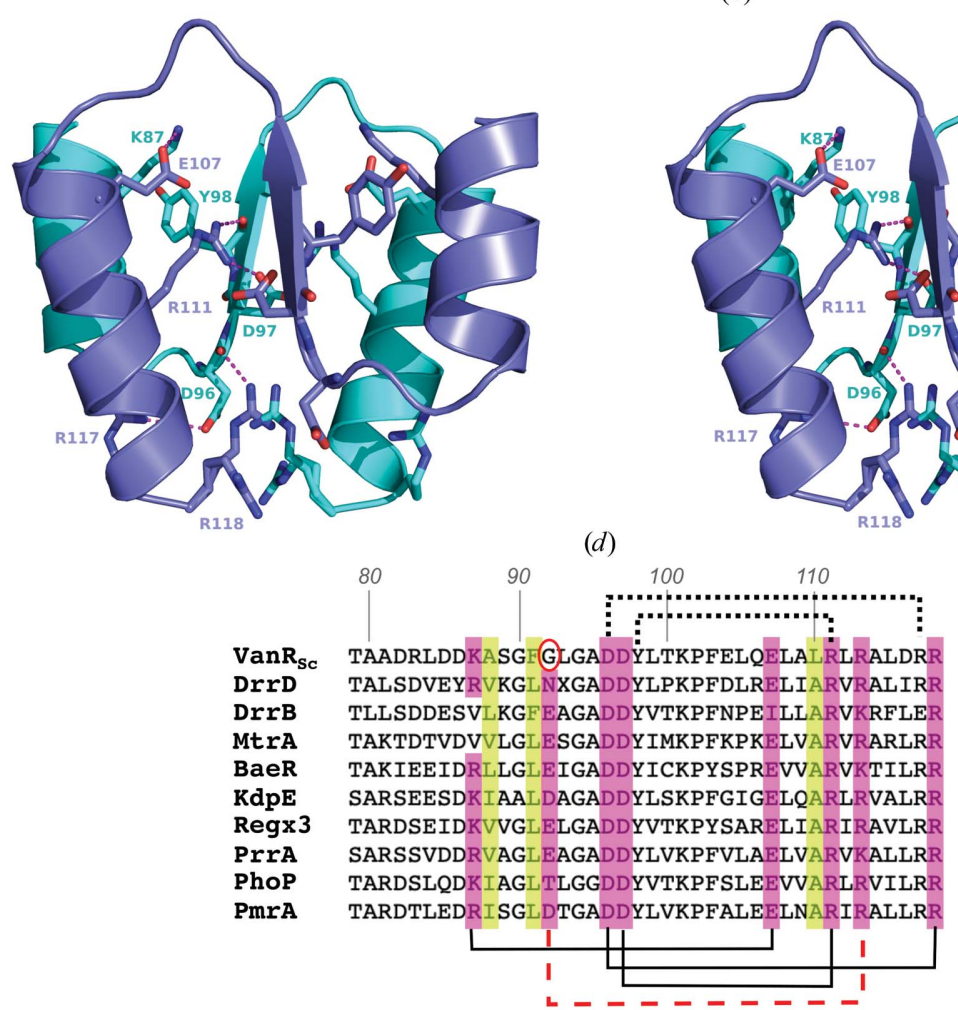

$(e)$

Figure 2

Activation of $\operatorname{VanR}_{\mathrm{Sc} \cdot}$ (a) A view from above the site of phosphorylation. The phosphoryl acceptor Asp51 lies at the end of the third $\beta$-strand and is shown bound to the beryllium fluoride phosphomimetic. The F atoms (pale blue) interact with the side chains of Thr79 and Lys101 and the backbone carbonyl of Asp53. A magnesium ion (shown in teal) is also present at the phosphorylation site, and is octahedrally coordinated by the side chains of Asp8, Glu7 and Asp51, the backbone carbonyl of Asp53 and two water molecules (shown as red spheres). (b) $2 F_{\mathrm{o}}-F_{\mathrm{c}}$ electron-density map showing the site of phosphorylation. A $\sigma_{\mathrm{A}}$-weighted map (Read, 1986) contoured at $1.6 \sigma$ is shown. A stereo version of this panel can be found in Supplementary Fig. S2. (c) The activated dimer, shown in both surface and cartoon representation. One protomer is colored cyan and the other is colored slate blue. The boxed region highlights the dimer interface that forms around $\alpha 4-\beta 5-\alpha 5$. Hydrophobic contacts along the outer edges of the dimer interface stabilize dimer formation; the amino-acid side chains responsible for these contacts are shown as spheres. $(d)$ Stereoview of polar contacts stabilizing the core of the dimer interface. The orientation shown is rotated $90^{\circ}$ about a horizontal axis relative to the orientation shown in $(c)$. (e) Conservation of dimerinterface residues for OmpR/PhoB response regulators. Hydrophobic residues are highlighted in lime green and polar residues are highlighted in pink. The brackets below the sequences indicate the typical salt bridges formed within the interface, while the brackets above indicate interactions that are specific to VanR $R_{S c}$. Because $V_{a n R}$ Sc contains a glycine at position 92 (circled), it does not form the typical 92-113 interaction (represented by the red dashed line); instead, an alternative hydrogen-bond interaction is formed between Tyr98 and Arg111. Numbering for the Van $R_{\mathrm{Sc}}$ sequence is shown at the top. For convenience, the brackets representing interactions are drawn connecting two residues within a single stretch of sequence; however, the actual interactions occur between two different protomers, across the dimer interface. 

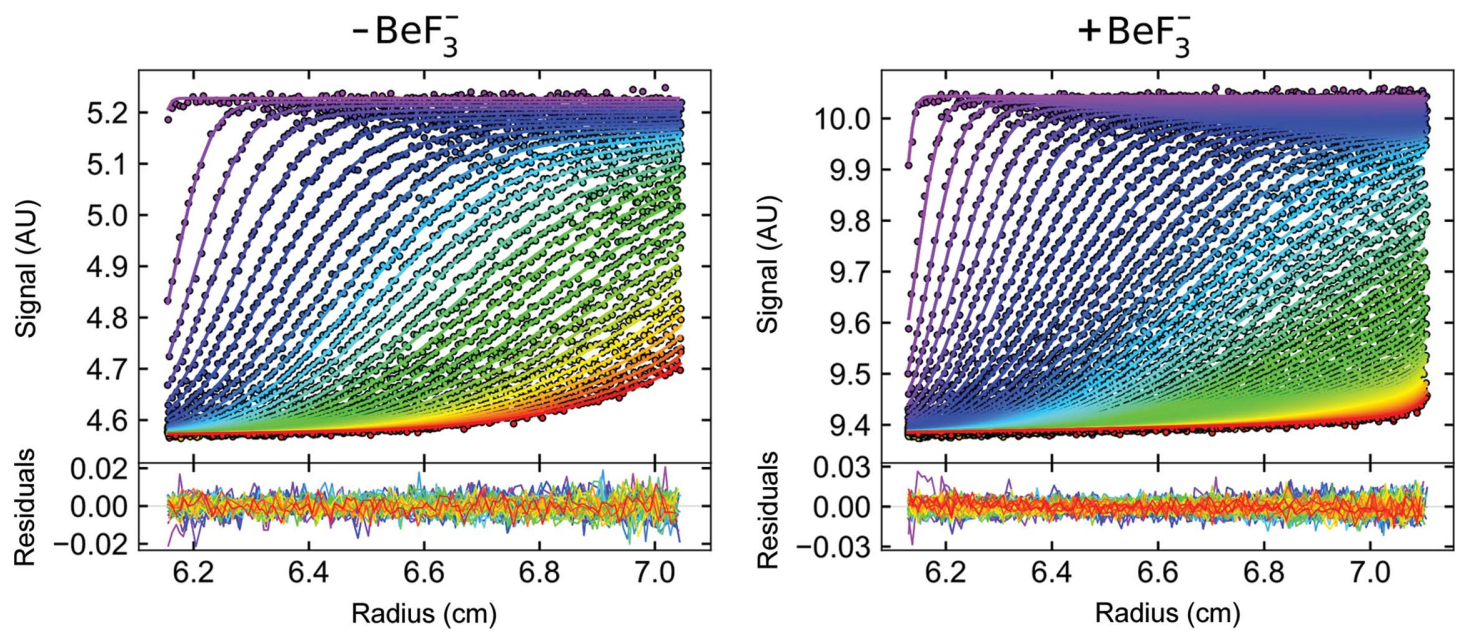

(a)

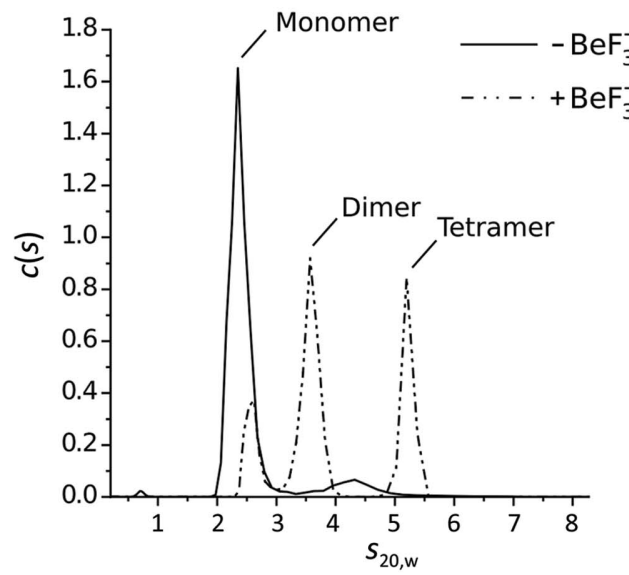

(b)
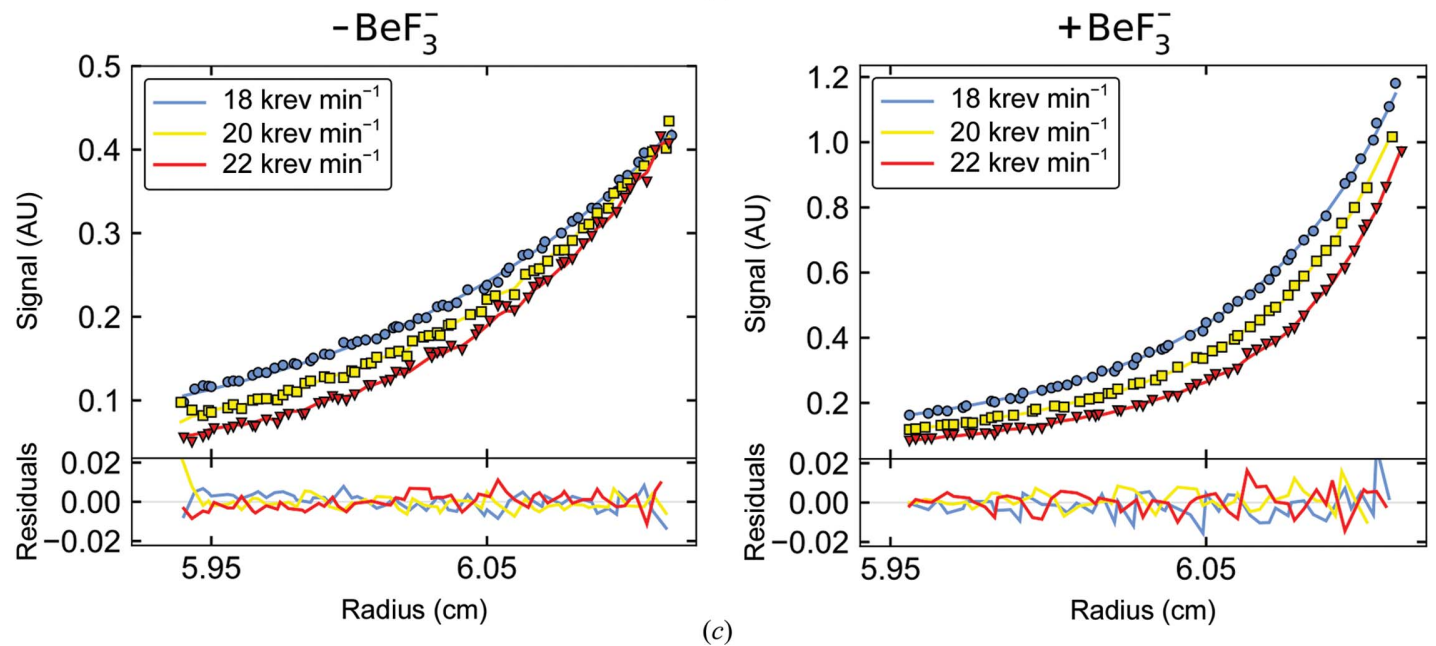

Figure 3

$\operatorname{VanR}_{\mathrm{Sc}}$ oligomerizes in the presence of beryllium fluoride. (a) Sedimentation-velocity analytical ultracentrifugation. Experimental data are shown as circles and fits of the Lamm equation are shown as lines; residuals from the fit are shown below the data panels. Only every third boundary and third data point are shown for clarity. Measurements were performed at a $36.5 \mu \mathrm{M}$ monomer concentration at $20^{\circ} \mathrm{C}$. $(b) c(s)$ distributions derived from the fitting of the Lamm equation to the data shown in $(a)$, as implemented in SEDFIT. The overall r.m.s.d. is 0.005 A for both fits. This analysis shows evidence of monomer plus small amounts of a larger species in the absence of $\mathrm{BeF}_{3}^{-}$, and monomers, dimers and tetramers in the presence of $\mathrm{BeF}_{3}^{-}$. These observations are consistent with the association constants derived from direct fitting of the sedimentation-velocity data to association models (see Supplementary Fig. S3 and Supplementary Table S3). (c) Sedimentation-equilibrium analytical ultracentrifugation. Representative data for $16.7 \mu M$ protein in the absence of $\mathrm{BeF}_{3}^{-}$(left) and for $47.3 \mu \mathrm{M}$ protein in the presence of $\mathrm{BeF}_{3}^{-}$(right) are shown. Model fits are shown as lines for each of three radial absorbance boundaries collected at three speeds $\left(18000,20000\right.$ and $\left.22000 \mathrm{rev} \mathrm{min}^{-1}\right)$; residuals for the model fitting are shown below the data panels. Data collected in the absence of $\mathrm{BeF}_{3}^{-}$are best described by a weak monomer-dimer equilibrium model consistent with the two species observed by sedimentation velocity; data collected in the presence of $\mathrm{BeF}_{3}^{-}$are best described by a monomer-dimer-tetramer equilibrium. Fit parameters are shown in Supplementary Table S4. Figures were prepared using GUSSI. 
consistent with the multiple species observed in the $c(s)$ distribution; estimated equilibrium dissociation constants for the two equilibria fall in the mid-micromolar range (Supplementary Table S3). The equilibrium data could also be fitted by a monomer-dimer-tetramer equilibrium model, providing a significantly better match than a monomer-dimer model. The dissociation constants for the equilibrium data were somewhat lower than those derived from the velocity data, as was seen for the data measured in the absence of $\mathrm{BeF}_{3}^{-}$ (Supplementary Table S4). However, both methods agree that the addition of $\mathrm{BeF}_{3}^{-}$is accompanied by a distinct shift from monomer to dimers and higher-order species.

\subsection{Comparison of the inactive and activated structures}

The receiver domains of $\operatorname{VanR}_{\mathrm{Sc}}$ in the inactive and activated states are extremely similar to each other, with an r.m.s.d. of $0.67 \AA$ for all $\mathrm{C}^{\alpha}$ atoms (Fig. $4 a$ ). The most noticeable difference between the two receiver domains is the absence of helix $\alpha 4$ in the inactive structure, along with significantly different conformations of the loop connecting $\beta 4$ to $\alpha 4$. In the inactive structure no electron density was observed for the entirety of $\alpha 4$, even though density is seen for the two flanking loops. To our knowledge, this level of $\alpha 4$ disorder has not been seen previously; however, this helix does adopt a range of different conformations in other response regulators, suggesting that conformational changes in $\alpha 4$ help to regulate activation (Buckler et al., 2002; Bachhawat et al., 2005; King-Scott et al., 2007; Choudhury \& Beis, 2013; see also PDB entry 3c97).

In addition to the gross changes surrounding $\alpha 4$, the two activity states exhibit smaller structural differences at the level of individual residues. One such difference is seen in the conserved amino-acid pair Thr79 and Tyr98. These correspond to the so-called switch residues, which favor different conformations in inactive versus activated receiver-domain structures (Gao et al., 2019). Phosphorylation of Asp51 drives the conformational equilibrium towards a structure in which the side chain of Thr79 has moved towards the active site and formed a hydrogen bond with an $\mathrm{O}$ atom of the phosphoryl group. At the same time, Tyr98 rotates its side chain upwards to fill the space that had been occupied by Thr79 (Fig. 5a). In this activated conformer, Tyr98 is stabilized by hydrogen bonding to the backbone carbonyl of Ala81 located in the $\beta 4$ $\alpha 4$ loop. It is not clear which inactive-state interactions, if any, are disrupted by this movement of Tyr98, since the lack of density for $\alpha 4$ prevents potential interactors from being identified. However, it is clear that in the inactive state $\alpha 4$ cannot occupy the same position that it does in the activated state, because the side chain of Tyr98 would clash with the helical backbone, as shown in Fig. 5(b).

$\operatorname{VanR}_{\mathrm{Sc}}$ does not form a dimer in the inactive state, and we reasoned that the transition from inactive monomer to activated dimer is likely to involve rearrangements of residues that create the $\alpha 4-\beta 5-\alpha 5$ dimer interface. After superposition of the inactive and activated structures, we analyzed the positions of the residues that form the interface. When going from the inactive to the activated state, the side chains of Arg111 and Arg118 rotate in order to interact with the backbone carbonyls of Tyr98 and Asp96, respectively. If Arg118 did not move in this way, it would clash with its symmetry mate in the other half of the dimer (Fig. 5c). Residues on $\alpha 4$ that contribute to the dimer interface (Ala88, Phe91 and Lys87) must also move in the shift from the inactive to the activated conformation, but the precise nature of these motions remains unknown, since $\alpha 4$ is disordered in the inactive state. However, it is likely that the interactions made by these three residues serve to stabilize $\alpha 4$ in its disorder-toorder transition.

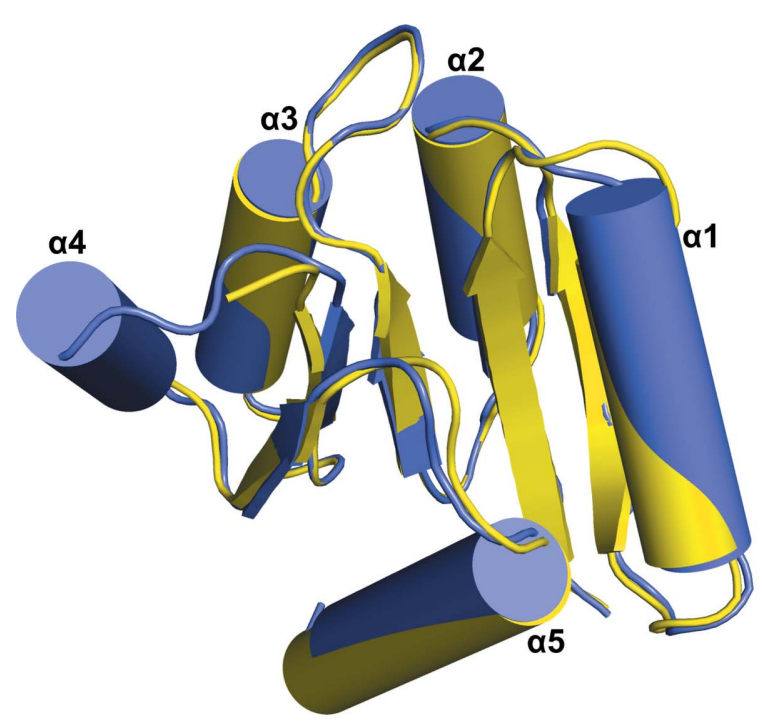

(a)

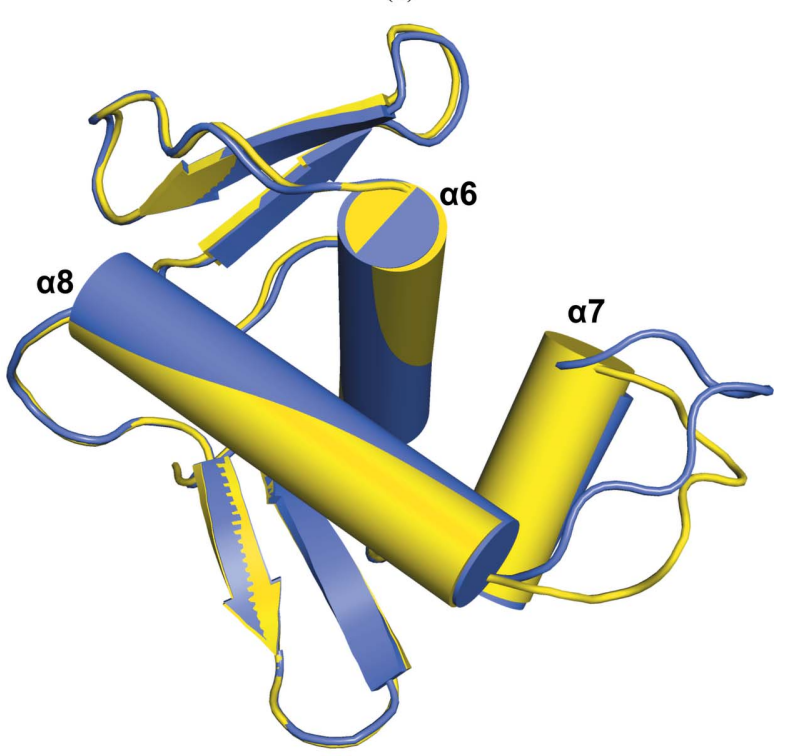

(b)

Figure 4

Domain comparison between the inactive and activated $V_{a n R}$ structures. Inactive $\operatorname{VanR}_{\mathrm{Sc}}$ is shown in yellow and activated $\operatorname{Van}_{\mathrm{Sc}}$ is shown in slate blue. (a) Superposition of the receiver domains; the largest differences are the lack of $\alpha 4$ in the inactive state and changes in conformation of the $\beta 4-\alpha 4$ loop. (b) Superposition of the DNA-binding domains; the only significant difference between these two structures is the conformation of the $\alpha 7-\alpha 8$ loop. 
Since activation of the response regulator promotes binding of its effector domain to its DNA target, we next compared the DNA-binding domains of $\operatorname{VanR}_{\mathrm{Sc}}$ in the inactive and activated states. The conformations of the DNA-binding domains are similar in these two activity states, with an r.m.s.d. of $0.96 \AA$ for all $\mathrm{C}^{\alpha}$ atoms (Fig. 4b). The most significant difference between
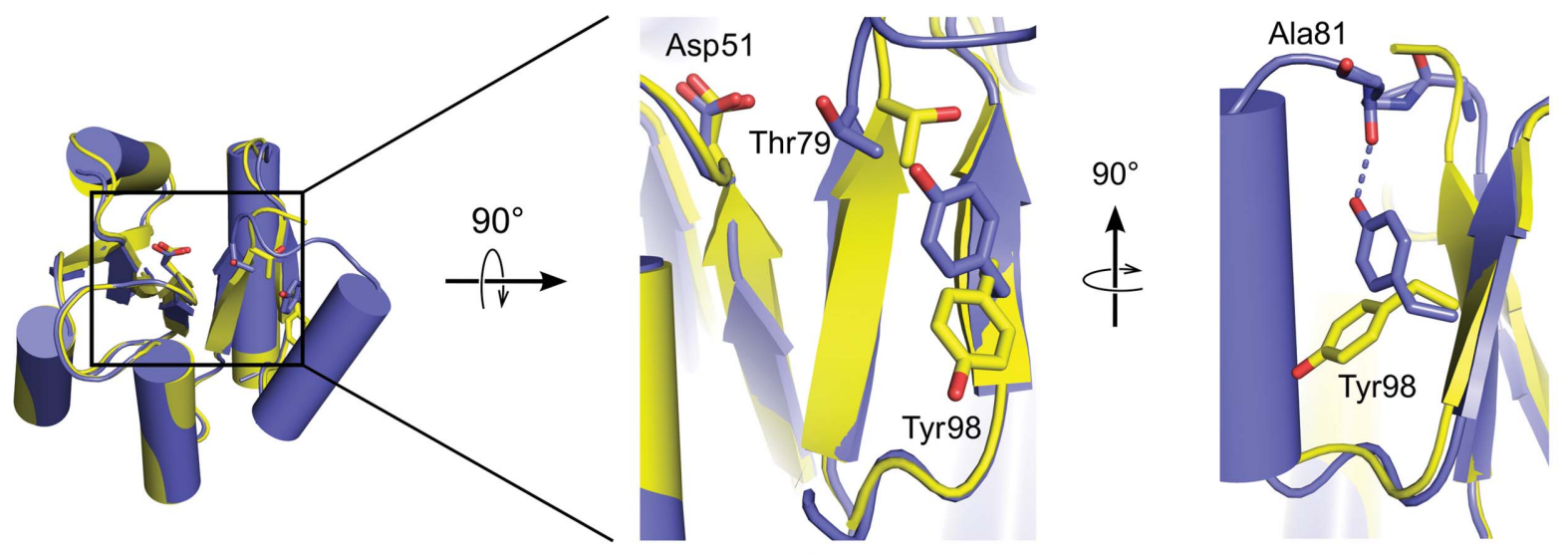

(a)
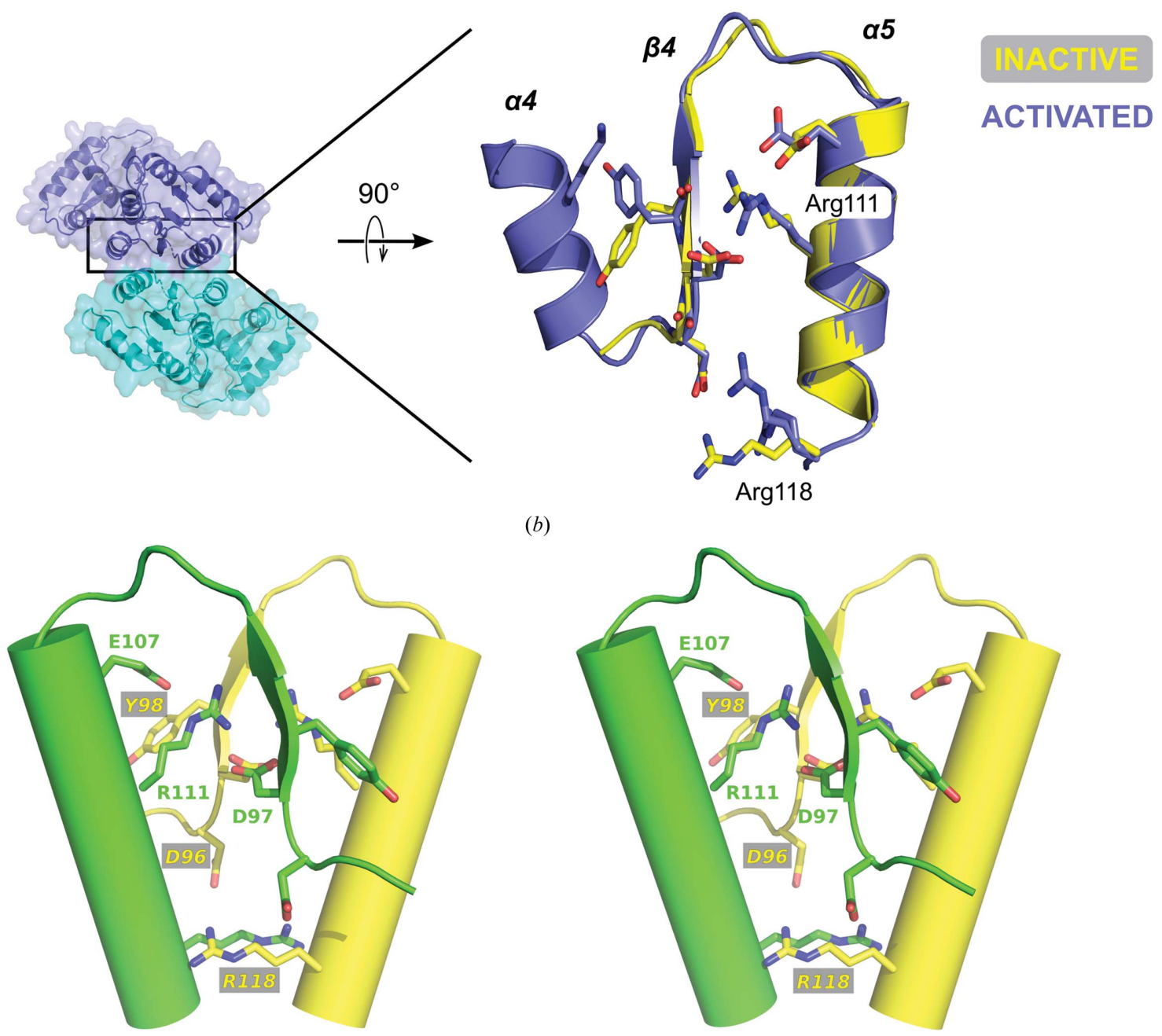

$(b)$

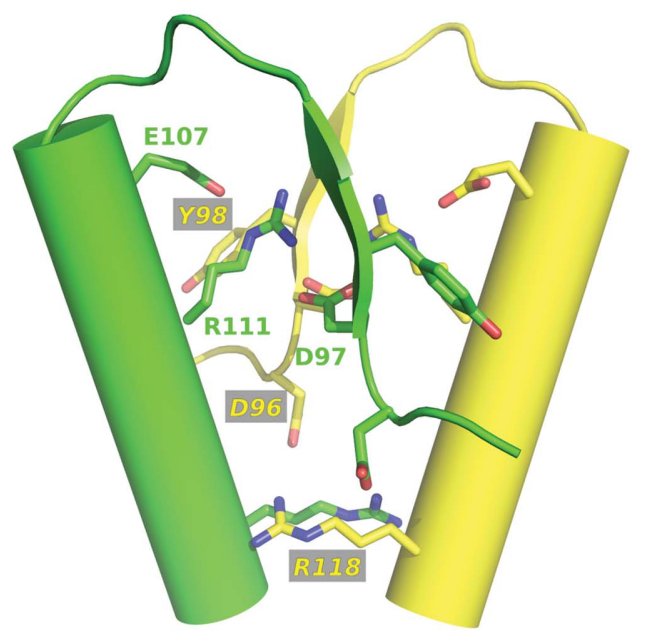

(c)

Figure 5

Structural changes associated with activation. (a) Changes in the switch residues. Inactive $V_{a n} R_{\mathrm{Sc}}$ is shown in yellow and activated Van $R_{\mathrm{Sc}}$ is shown in slate blue. (b) Superposition of $\alpha 4-\beta 5-\alpha 5$ in the inactive and activated states highlights the side-chain movements of polar interface residues, notably Arg111 and Arg118. (c) Stereoview of a mock dimer interface produced by superposing the inactive structure onto the activated structure. The resulting two inactive-state protomers are shown in green and yellow. Side-chain conformations in the inactive state are incompatible with the activated-state dimer interface: Arg111 and Tyr98 are too far apart to interact, as are Arg118 and Asp96. Additionally, the inactive-state conformation of Arg118 would clash with its symmetry mate. 
the two conformations is the position of the $\alpha 7-\alpha 8$ loop. The conformation of this loop varies substantially in different response-regulator structures, which may reflect an inherent flexibility that allows optimal fitting at the protein-DNA interface (Robinson et al., 2003).

\subsection{Comparison of activated $\operatorname{Van} R_{\mathrm{Sc}}$ with other activated OmpR/PhoB response regulators}

Many activated OmpR/PhoB response regulators (for example, KdpE and PmrA) assemble onto DNA with their DNA-binding domains arranged in a head-to-tail manner (Narayanan et al., 2014; Lou et al., 2015). Others (for example,
OmpR) are able to bind in either a head-to-tail or a head-tohead orientation depending upon the specific sequences of the recognition sites (Maris et al., 2005; Rhee et al., 2008). However, in the dimer of activated $\operatorname{VanR}_{\mathrm{Sc}}$ the two DNAbinding domains are positioned far from each other and adopt neither a head-to-tail nor a head-to-head orientation (Fig. 6a). This difference in the positioning of the DNA-binding domains does not result from differences in the receiver domain, since KdpE and PmrA both form $\alpha 4-\beta 5-\alpha 5$ dimers that are similar to the $\operatorname{VanR}_{\mathrm{Sc}}$ dimer. Presumably, therefore, in the presence of DNA, the DNA-binding domain of $V_{\mathrm{San}}$ rearrange themselves so as to assemble head to tail. Such a rearrangement seems plausible, given the long linker
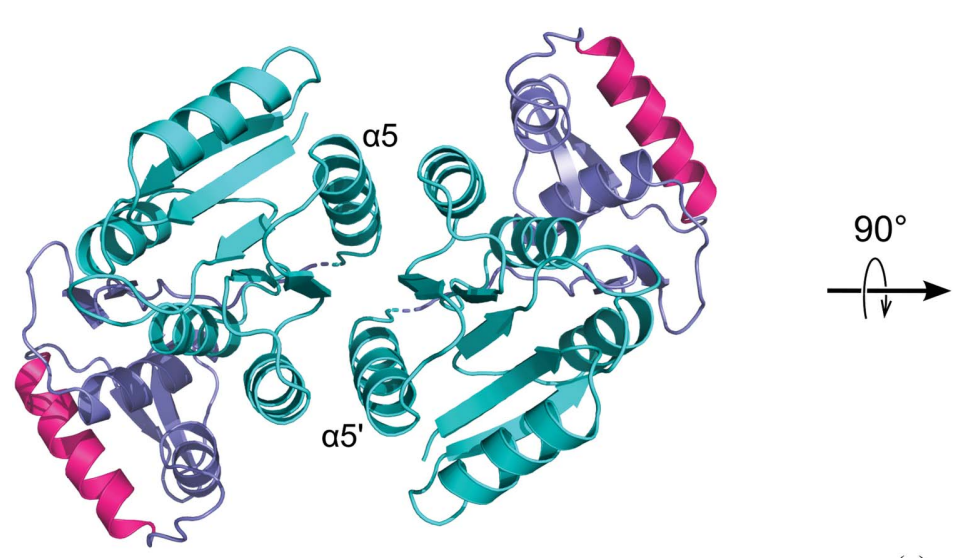

(a)

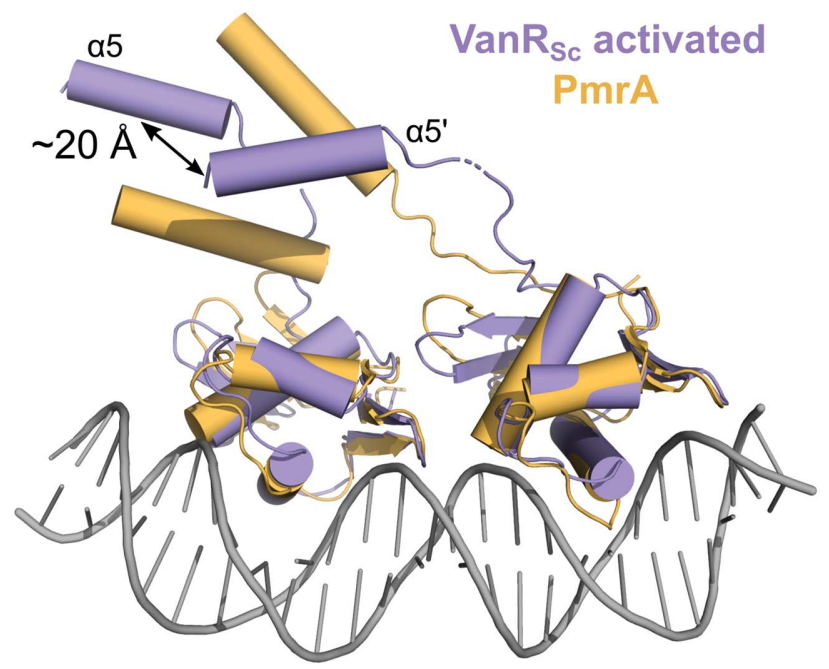

(b)
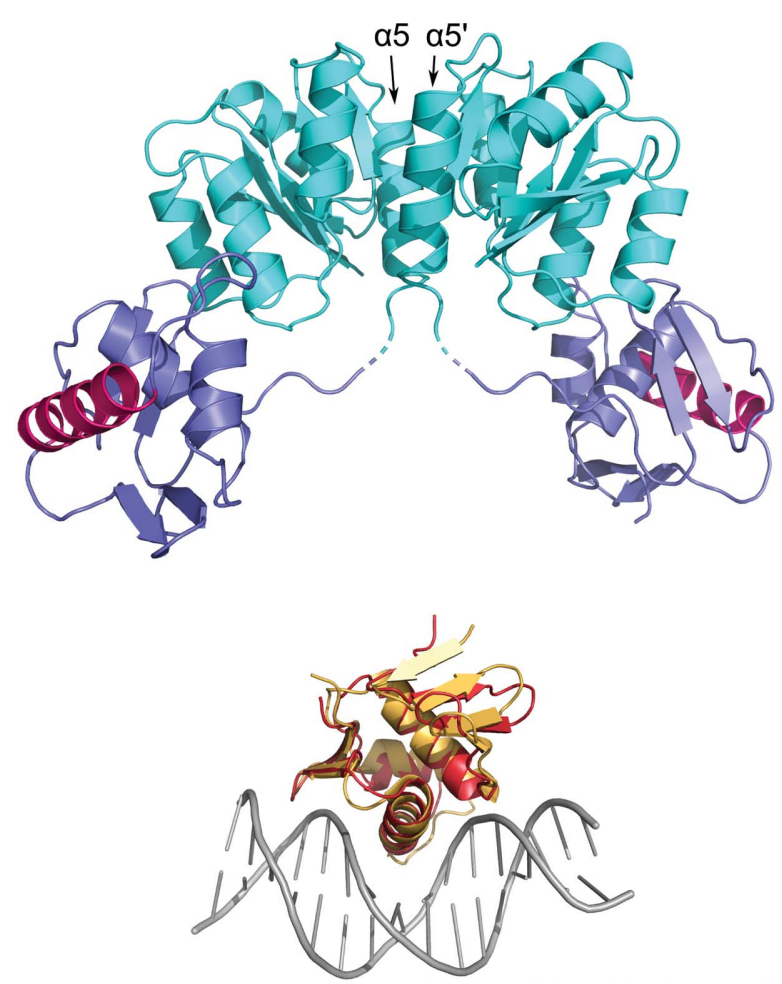

(c)

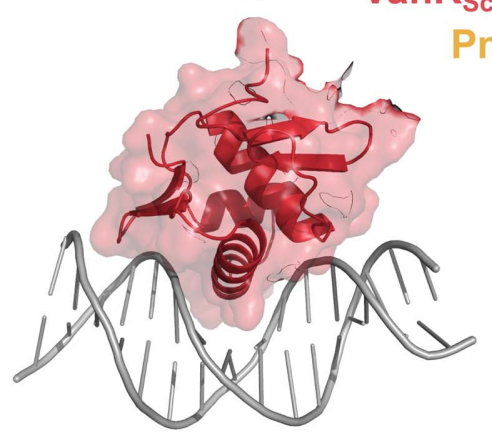

(d)

Figure 6

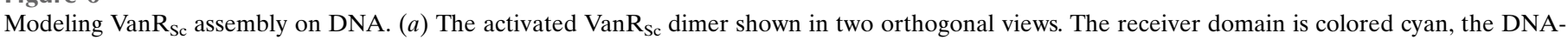

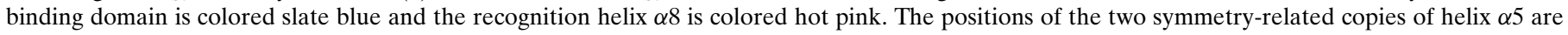

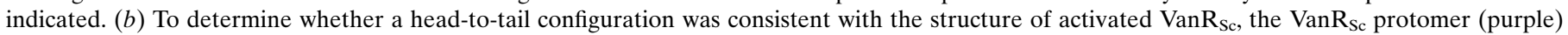

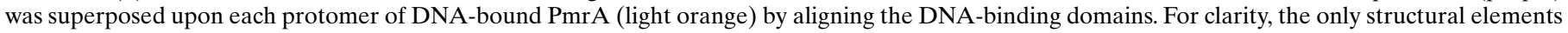

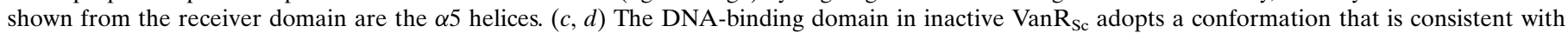

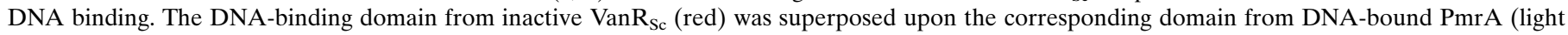

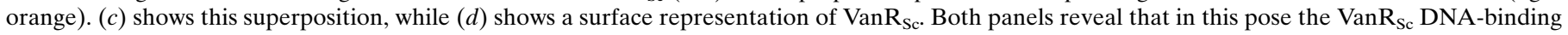
domain does not clash with the DNA. The PmrA structure was taken from PDB entry $4 \mathrm{~s} 04$. 
connecting the receiver and DNA-binding domains of $\operatorname{VanR}_{\mathrm{Sc}}$. This linker is approximately $35 \AA$ in length, which is substantially longer than the linkers in KdpE and PmrA (19 and $30 \AA$, respectively). To test the plausibility of such a rearrangement, the DNA-binding domains of two copies of activated $V_{a n R} R_{S c}$ were superposed on each of the two corresponding domains of the DNA-bound PmrA structure. Placing the two $\operatorname{VanR}_{\mathrm{Sc}}$ monomers into this head-to-tail arrangement resulted in their $\alpha 5$ helices being positioned roughly parallel to one another, with the helix axes offset by approximately $20 \AA$ (Fig. 6b). The relative positions of these two helices are close to what is seen in the actual receiver-domain dimer, where these two helices are also roughly parallel to one another, with their axes separated by $\sim 14 \AA$ at one end of the helices and $\sim 22 \AA$ at the other (Fig. 6a). Therefore, small adjustments in the flexible linkers should be sufficient to allow the activated $V_{a n R}$ Sc protein to form the canonical receiver-domain dimer, while at the same time allowing its DNA-binding domains to bind the target DNA in the expected head-to-tail conformation. We must note, however, that the precise DNA sequences recognized by $V_{a n R_{S c}}$ are not yet known: while the corresponding recognition sequences are known for the VanR proteins from type A and type B VRE, these proteins share less than $20 \%$ sequence identity with $V_{a n} R_{S c}$, and the upstream regions containing VanR sites are similarly divergent. Thus, in the absence of detailed knowledge about the recognition sites of $V a n R_{S c}$, the relative positioning of the two protomers when bound to DNA remains a point of speculation.

\subsection{Comparison of inactive $\operatorname{Van}_{\mathrm{Sc}}$ with other inactive OmpR/PhoB response regulators}

Why is unphosphorylated $\operatorname{VanR}_{\mathrm{Sc}}$ inactive? The DNAbinding domain changes very little between the inactive and activated states, and even in the inactive state it adopts a conformation that appears competent to bind DNA (Figs. $6 c$ and $6 d$ ). To address the molecular basis for inactivation, it is useful to consider inactive-state structures of other OmpR/ PhoB-family response regulators. These structures suggest that more than one regulatory mechanism exists. For example, in PrrA and MtrA the DNA-binding domain is attached to the receiver domain so as to occlude the recognition helix, thereby preventing DNA binding (Fig. 7a; Nowak et al., 2006; Friedland et al., 2007). In contrast, in DrrD and DrrB the recognition helix is not occluded in the inactive state; however, the two domains interact in a way that is thought to limit the mobility of the DNA-binding domain and thus hinder DNA binding (Buckler et al., 2002; Robinson et al., 2003). Finally, in addition to the inactivation mechanisms suggested by the structures described above, an additional possible mechanism is suggested by the observation that linker length and

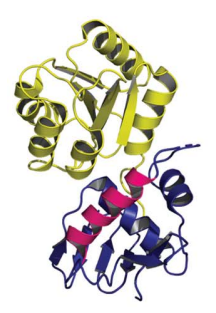

MtrA

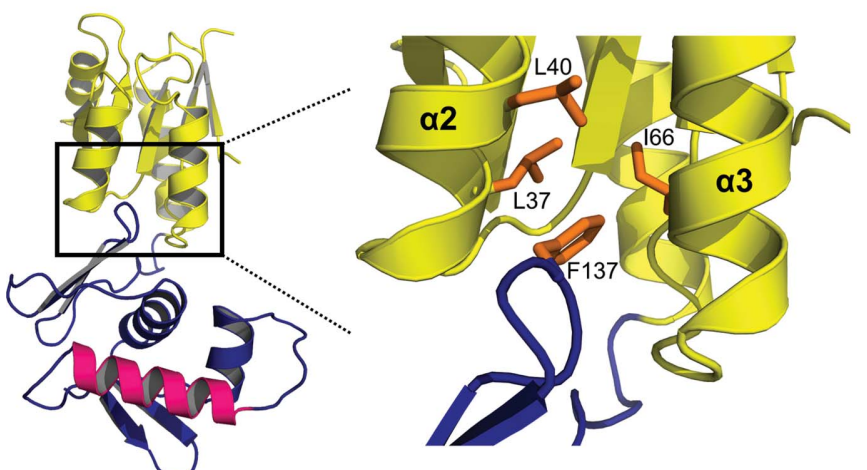

(b)

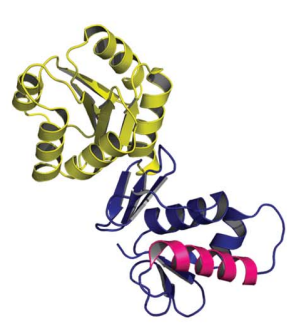

DrrB

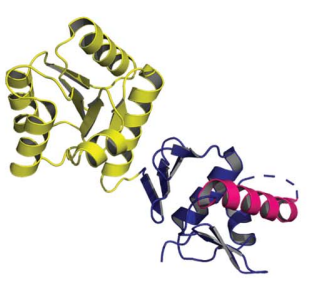

DrrD

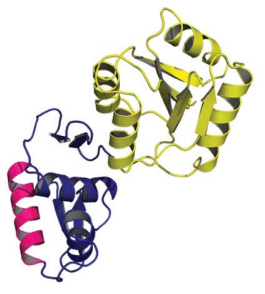

$\operatorname{VanR}_{\mathrm{Sc}}$-inactive

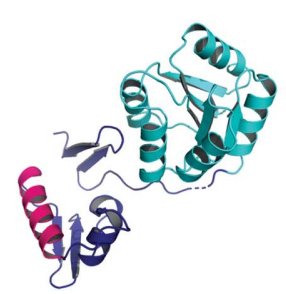

VanR $_{\text {Sc }}$-activated

(a)
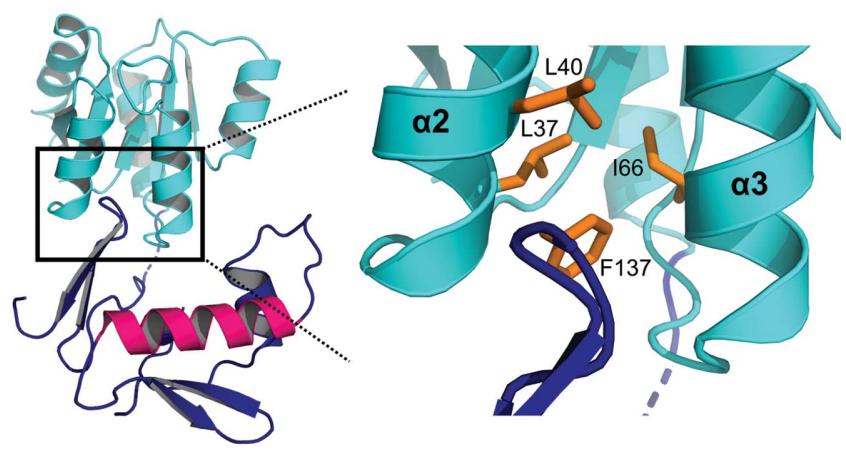

(c)

Figure 7

Modes of inactivation for OmpR/PhoB response regulators. (a) Inactive conformations of four different response regulators are shown, compared with the inactive conformation of $\operatorname{VanR}_{\mathrm{Sc}}$. For the inactive response regulators, the receiver domains are shown in yellow, the DNA-binding domains in dark blue and the recognition helix $\alpha 8$ in hot pink. The activated $V_{a n R_{S c}}$ structure is also shown for the sake of comparison, with the receiver domain colored cyan. This panel was inspired by a figure in Friedland et al. (2007). PDB codes are as follows: MtrA, 2gwr; PrrA, 1ys6; DrrB, 1p2f; DrrD, 1kgs. (b) In the inactive $\operatorname{VanR}_{\mathrm{Sc}}$ structure, the interdomain interaction involves the insertion of the $\beta 6-\beta 7$ turn from the DNA-binding domain into a cleft between helices $\alpha 2$ and $\alpha 3$ of the receiver domain, placing Phe137 into a hydrophobic pocket formed by Leu37, Leu40 and Ile66. (c) In the activated VanR $\mathrm{Sc}$ structure a similar interdomain interaction is also formed, despite a change in the relative orientations of the two domains. 
composition can alter response-regulator function (Mattison et al., 2002; Walthers et al., 2003); such linker effects may manifest dynamically, altering the relative mobility of the receiver and DNA-binding domains, and as such may prove difficult to capture structurally.

In the inactive $\operatorname{VanR}_{\mathrm{sc}}$ structure, the receiver and DNAbinding domains also interact via a small interdomain interface that is formed by the insertion of a $\beta$-turn from the DNAbinding domain into a cleft in the receiver domain, in an interaction that buries $260 \AA^{2}$ of surface area (Fig. 7b). This places the side chain of Phe137 into a hydrophobic pocket between helices $\alpha 2$ and $\alpha 3$, lined by the side chains of Leu37, Leu40 and Ile66. This interdomain interface is distinct from that seen in the PrrA and MtrA structures and does not occlude the recognition helix (Fig. 7a). Interestingly, a similar interdomain interaction occurs in the structure of activated $V_{a n R}$, despite the fact that the domains change their relative orientations in the inactive versus activated structures (Fig. 7c). Given that this interdomain interaction appears to be able to accommodate some conformational variation, we suggest that a DNA-binding domain from one dimer might be able to interact intermolecularly with the receiver domain from another dimer, providing a potential explanation for the putative tetrameric species seen in the ultracentrifugation experiments. Even if this conjecture is correct, however, the biological relevance of oligomers larger than dimers remains unclear.

If the interdomain interaction does block activation by immobilizing the DNA-binding domain, as has been suggested for DrrD and DrrB, the interaction must be substantially stronger in the inactive state than in the activated state. This appears unlikely, given the similarity between the interdomain interfaces in the two states. Hence, we speculate that this interaction is sufficiently weak that it can be readily disrupted in the presence of the DNA target, freeing the DNA-binding domains so they may optimally orient themselves on the target. In conclusion, the structural evidence suggests that neither occlusion of the recognition helix nor immobilization of the DNA-binding domain is responsible for maintaining $V_{a n R}$ in an inactive state.

In the structure of inactive $\operatorname{VanR}_{\mathrm{sc}}$, the linker connecting the receiver and DNA-binding domains is $27 \AA$ in length, which presumably allows the DNA-binding domain to sample many different positions and orientations. This, together with the accessibility of the recognition helix, suggests that inactive VanR $R_{\mathrm{Sc}}$ should be able to bind DNA. In support of this notion, the DNA-binding domain adopts a conformation that is compatible with target binding; for example, it can be superposed onto the DNA-bound structure of PmrA without clashing with the DNA (Figs. $6 c$ and $6 d$ ). Indeed, the VanR orthologs from A- and B-type VRE have been shown to bind DNA in their unphosphorylated states, albeit much more weakly than the phosphorylated proteins (Holman et al., 1994; Depardieu et al., 2005). Therefore, we suggest that no structural impediment prevents $V_{a n R_{S c}}$ from binding DNA in its inactive state; however, this binding will be weak in the absence of dimerization. Once the protein is phosphorylated, the dimerization induced by the activating signal will enhance DNA binding through an avidity effect.

\section{Conclusions}

We present here the first full-length $\mathrm{OmpR} / \mathrm{PhoB}$ response regulator to be crystallized in both the inactive and activated states. The main structural differences between the two activity states center around the stability of $\alpha 4$ and the oligomeric state of the protein. Upon phosphorylation of Asp51, $\alpha 4$ transitions from a disordered to an ordered state, stabilizing key residues involved in forming the $\alpha 4-\beta 5-\alpha 5$ dimer interface. We propose that this phosphorylationinduced dimerization provides an avidity effect that enhances DNA binding and promotes transcription. Overall, these structures suggest that the key feature required for the activation of transcription by $\operatorname{VanR}_{\mathrm{Sc}}$ is its dimerization.

\section{Acknowledgements}

This research used the AMX beamline (17-ID-1) of the National Synchrotron Light Source II, a US Department of Energy (DOE) Office of Science User Facility operated for the DOE Office of Science by Brookhaven National Laboratory under Contract No. DE-SC0012704. The AMX facility is part of the Center for BioMolecular Structure (CBMS), which is primarily supported by the National Institutes of Health, National Institute of General Medical Sciences (NIGMS) through a Center Core P30 Grant (P30GM133893), and by the DOE Office of Biological and Environmental Research (KP1605010). Initial diffraction experiments were conducted on the Northeastern Collaborative Access Team beamlines, which are funded by the National Institute of General Medical Sciences from the National Institutes of Health (P30 GM124165). The EIGER 16M detector on the 24-ID-E beamline is funded by an NIH-ORIP HEI grant (S10OD021527). This research used resources of the Advanced Photon Source, a US Department of Energy (DOE) Office of Science User Facility operated for the DOE Office of Science by Argonne National Laboratory under Contract No. DE-AC02-06CH11357. Analytical ultracentrifugation analyses were performed at the Johnson Foundation Structural Biology and Biophysics Core at the Perelman School of Medicine, Philadelphia, Pennsylvania with the support of an NIH High-End Instrumentation Grant (S10OD018483).

\section{Funding information}

We gratefully acknowledge funding from the National Institutes of Health, National Institute of Allergy and Infectious Diseases, including grants F31 AI136385 (LJM) and R01 AI148679 (PJL).

\section{References}

Arthur, M., Molinas, C. \& Courvalin, P. (1992). J. Bacteriol. 174, 25822591. 
Bachhawat, P., Swapna, G. V., Montelione, G. T. \& Stock, A. M. (2005). Structure, 13, 1353-1363.

Bentley, S. D., Chater, K. F., Cerdeño-Tárraga, A. M., Challis, G. L., Thomson, N. R., James, K. D., Harris, D. E., Quail, M. A., Kieser, H., Harper, D., Bateman, A., Brown, S., Chandra, G., Chen, C. W., Collins, M., Cronin, A., Fraser, A., Goble, A., Hidalgo, J., Hornsby, T., Howarth, S., Huang, C. H., Kieser, T., Larke, L., Murphy, L., Oliver, K., O'Neil, S., Rabbinowitsch, E., Rajandream, M. A., Rutherford, K., Rutter, S., Seeger, K., Saunders, D., Sharp, S., Squares, R., Squares, S., Taylor, K., Warren, T., Wietzorrek, A., Woodward, J., Barrell, B. G., Parkhill, J. \& Hopwood, D. A. (2002). Nature, 417, 141-147.

Blanco, A. G., Sola, M., Gomis-Rüth, F. X. \& Coll, M. (2002). Structure, 10, 701-713.

Brautigam, C. A. (2015). Methods Enzymol. 562, 109-133.

Buckler, D. R., Zhou, Y. \& Stock, A. M. (2002). Structure, 10, $153-$ 164.

Chayen, N. E., Shaw Stewart, P. D. \& Blow, D. M. (1992). J. Cryst. Growth, 122, 176-180.

Chen, V. B., Arendall, W. B., Headd, J. J., Keedy, D. A., Immormino, R. M., Kapral, G. J., Murray, L. W., Richardson, J. S. \& Richardson, D. C. (2010). Acta Cryst. D66, 12-21.

Choudhury, H. G. \& Beis, K. (2013). Protein Sci. 22, 1287-1293.

D'Arcy, A., Elmore, C., Stihle, M. \& Johnston, J. E. (1996). J. Cryst. Growth, 168, 175-180.

Depardieu, F., Courvalin, P. \& Kolb, A. (2005). Mol. Microbiol. 57, $550-564$.

Diederichs, K. \& Karplus, P. A. (1997). Nat. Struct. Mol. Biol. 4, 269 275.

Emsley, P., Lohkamp, B., Scott, W. G. \& Cowtan, K. (2010). Acta Cryst. D66, 486-501.

Evers, S. \& Courvalin, P. (1996). J. Bacteriol. 178, 1302-1309.

Friedland, N., Mack, T. R., Yu, M., Hung, L. W., Terwilliger, T. C., Waldo, G. S. \& Stock, A. M. (2007). Biochemistry, 46, 6733-6743.

Galperin, M. Y. (2006). J. Bacteriol. 188, 4169-4182.

Gao, R., Bouillet, S. \& Stock, A. M. (2019). Annu. Rev. Microbiol. 73, 175-197.

He, X., Wang, L. \& Wang, S. (2016). Sci. Rep. 6, 24442.

Holman, T. R., Wu, Z., Wanner, B. L. \& Walsh, C. T. (1994). Biochemistry, 33, 4625-4631.

Hong, H. J., Hutchings, M. I. \& Buttner, M. J. (2008). Adv. Exp. Med. Biol. 631, 200-213.

Janin, J., Rodier, F., Chakrabarti, P. \& Bahadur, R. P. (2007). Acta Cryst. D63, 1-8.

Kabsch, W. (2010). Acta Cryst. D66, 125-132.

Karplus, P. A. \& Diederichs, K. (2012). Science, 336, 1030-1033.

King-Scott, J., Nowak, E., Mylonas, E., Panjikar, S., Roessle, M., Svergun, D. I. \& Tucker, P. A. (2007). J. Biol. Chem. 282, 3771737729.

Krissinel, E. \& Henrick, K. (2007). J. Mol. Biol. 372, 774-797.

Kumar, P. \& Bansal, M. (2012). J. Biomol. Struct. Dyn. 30, 773-783.

Laue, T. M., Shah, B. D., Ridgeway, T. M. \& Pelletier, S. L. (1992). Ultracentrifugation in Biochemistry and Polymer Science, edited by S. Harding, A. Rowe \& J. Horton, pp. 90-125. Cambridge: Royal Society of Chemistry.

Lee, B. \& Richards, F. M. (1971). J. Mol. Biol. 55, 379-400.

Liebschner, D., Afonine, P. V., Baker, M. L., Bunkóczi, G., Chen, V. B., Croll, T. I., Hintze, B., Hung, L.-W., Jain, S., McCoy, A. J., Moriarty, N. W., Oeffner, R. D., Poon, B. K., Prisant, M. G., Read, R. J., Richardson, J. S., Richardson, D. C., Sammito, M. D., Sobolev, O. V., Stockwell, D. H., Terwilliger, T. C., Urzhumtsev, A. G.,
Videau, L. L., Williams, C. J. \& Adams, P. D. (2019). Acta Cryst. D75, 861-877.

Lou, Y.-C., Weng, T.-H., Li, Y.-C., Kao, Y.-F., Lin, W.-F., Peng, H.-L., Chou, S.-H., Hsiao, C.-D. \& Chen, C. (2015). Nat. Commun. 6, 8838.

Maris, A. E., Walthers, D., Mattison, K., Byers, N. \& Kenney, L. J. (2005). J. Mol. Biol. 350, 843-856.

Martínez-Hackert, E. \& Stock, A. M. (1997). J. Mol. Biol. 269, 301312.

Mattison, K., Oropeza, R. \& Kenney, L. J. (2002). J. Biol. Chem. 277, 32714-32721.

Milton, M. E., Allen, C. L., Feldmann, E. A., Bobay, B. G., Jung, D. K., Stephens, M. D., Melander, R. J., Theisen, K. E., Zeng, D., Thompson, R. J., Melander, C. \& Cavanagh, J. (2017). Mol. Microbiol. 106, 223-235.

Narayanan, A., Kumar, S., Evrard, A. N., Paul, L. N. \& Yernool, D. A. (2014). Nat. Commun. 5, 3282.

Nguyen, M.-P., Yoon, J.-M., Cho, M.-H. \& Lee, S.-W. (2015). Can. J. Microbiol. 61, 799-810.

Nowak, E., Panjikar, S., Konarev, P., Svergun, D. I. \& Tucker, P. A. (2006). J. Biol. Chem. 281, 9659-9666.

Ponstingl, H., Henrick, K. \& Thornton, J. M. (2000). Proteins, 41, 4757.

Read, R. J. (1986). Acta Cryst. A42, 140-149.

Rhee, J. E., Sheng, W., Morgan, L. K., Nolet, R., Liao, X. \& Kenney, L. J. (2008). J. Biol. Chem. 283, 8664-8677.

Robinson, V. L., Buckler, D. R. \& Stock, A. M. (2000). Nat. Struct. Biol. 7, 626-633.

Robinson, V. L., Wu, T. \& Stock, A. M. (2003). J. Bacteriol. 185, 41864194.

Saff, E. B. \& Kuijlaars, A. B. J. (1997). Math. Intelligencer, 19, 5-11.

Schuck, P. (2000). Biophys. J. 78, 1606-1619.

Stock, A. M., Mottonen, J. M., Stock, J. B. \& Schutt, C. E. (1989). Nature, 337, 745-749.

Toro-Roman, A., Mack, T. R. \& Stock, A. M. (2005). J. Mol. Biol. 349, $11-26$.

Toro-Roman, A., Wu, T. \& Stock, A. M. (2005). Protein Sci. 14, 3077 3088.

Vistica, J., Dam, J., Balbo, A., Yikilmaz, E., Mariuzza, R. A., Rouault, T. A. \& Schuck, P. (2004). Anal. Biochem. 326, 234-256.

Walthers, D., Tran, V. K. \& Kenney, L. J. (2003). J. Bacteriol. 185, $317-$ 324.

Wang, S., Engohang-Ndong, J. \& Smith, I. (2007). Biochemistry, 46, 14751-14761.

Weeks, S. D., Drinker, M. \& Loll, P. J. (2007). Protein Expr. Purif. 53, 40-50.

Weiss, M. S. (2001). J. Appl. Cryst. 34, 130-135.

Wemmer, D. E. \& Kern, D. (2005). J. Bacteriol. 187, 8229-8230.

West, A. H. \& Stock, A. M. (2001). Trends Biochem. Sci. 26, 369-376.

Winn, M. D., Ballard, C. C., Cowtan, K. D., Dodson, E. J., Emsley, P., Evans, P. R., Keegan, R. M., Krissinel, E. B., Leslie, A. G. W., McCoy, A., McNicholas, S. J., Murshudov, G. N., Pannu, N. S., Potterton, E. A., Powell, H. R., Read, R. J., Vagin, A. \& Wilson, K. S. (2011). Acta Cryst. D67, 235-242.

Yan, D., Cho, H. S., Hastings, C. A., Igo, M. M., Lee, S. Y., Pelton, J. G., Stewart, V., Wemmer, D. E. \& Kustu, S. (1999). Proc. Natl Acad. Sci. USA, 96, 14789-14794.

Zhang, Y. \& Skolnick, J. (2005). Nucleic Acids Res. 33, 2302-2309.

Zwart, P. H., Afonine, P. V., Grosse-Kunstleve, R. W., Hung, L. W., Ioerger, T. R., McCoy, A. J., McKee, E., Moriarty, N. W., Read, R. J., Sacchettini, J. C., Sauter, N. K., Storoni, L. C., Terwilliger, T. C. \& Adams, P. D. (2008). Methods Mol. Biol. 426, 419-435. 